\section{Experiments in the gauge revolution}

\section{C.H. Llewellyn Smith}

Weak Interactions of Leptons and

Quarks.

By E.D. Commins and

P.H. Bucksbaum.

Cambridge University Press: 1983.

Pp.473. Hbk £37.50, \$69.50;

pbk £16.50, \$27.95.

FOLLOWING the discovery of parity violation in $\beta$ decay in 1957, it was soon found that a wide range of "weak" decay processes can be described by a simple " "phenomenological"' theory. The pejorative adjective indicates that this theory is not mathematically consistent and can only work in a limited energy domain. In 1971, 't Hooft showed that consistency could be obtained in gauge theories of the type discussed earlier by Weinberg, Salam, Glashow and others, which have the great attraction of unifying weak and electromagnetic interactions. There was then no experimental evidence for such theories but their revolutionary potential was sufficient to stem the flow of books on weak interactions which had appeared during the 1960 s.

Confidence in gauge theories built up as their predictions were gradually confirmed, beginning with the discovery of "neutral current" neutrino interactions at CERN in 1973 and culminating in the discovery of the $\mathrm{W}$ and $\mathrm{Z}$ bosons - the carriers of the weak force - with the predicted masses at CERN last year. Reflecting these developments, textbooks which incorporate or describe the "gauge revolution" have begun to appear in the past few years. This one, which is suitable for graduate students or as a reference work for researchers, is the first of the new generation of books written from an experimental standpoint in which crucial experiments are described. As well as the traditional topics, there are chapters on most of the subjects which ought now to be in such books - high-energy neutrino interactions, neutrino masses and oscillations, parity non-conservation in $\mathrm{eN}$ and $\mathrm{NN}$ interactions (including in atoms - the authors' speciality) and neutrinos in astrophysics.

Turning to omissions, it is odd that there is no derivation - or even statement - of the Fierz transformation, although it is frequently used. The omission of a proper discussion of current algebra and chiral symmetry is more serious. The AdlerWeisberger and Goldberger-Treiman relations appear (although the latter is confused with pole dominance of the pseudo-scalar form factor and we are not told whether the real relation works). Discussion of the Callan-Treiman relation and predictions for slope parameters in $\mathrm{K}$ $\rightarrow 3 \pi$ surely also deserved some space. On the other hand, the "abbreviated and superficial" discussion of path integrals will help neither the novice nor the expert and could have been left out. The authors nowhere really use or need the full Feynman rules which they derive using this technique (although some discussion of testable higher-order predictions of gauge theories might have been desirable).

A more serious criticism is that the book contains several theoretical remarks which are dubious or misleading. Two of the more subtle examples must suffice. It is not true that Lagrangians "satisfy the Euler Lagrange equations" or that current conservation is in general equivalent to invariance of the Lagrangian (the whole question of auxiliary fields in supersymmetry bears witness to this). The massmatrix for the $\mathbf{K}^{0}-\overline{\mathbf{K}}^{0}$ system does not follow from the Schrodinger equation given (although the appearance of the word "effective" perhaps provides an alibi) - a derivation involves the Wigner-Weisskopf

\section{Exciting stuff}

\section{F.W.J. Teale}

\section{Principles of Fluorescence}

Spectroscopy.

By Joseph R. Lakowicz.

Plenum: 1983. Pp.496. \$32.50, £25.

EVEN the most cursory glance over the literature of biochemistry, biotechnology and clinical medicine confirms the continuing growth in the exploitation of fluorescence techniques. How then should uninitiated researchers equip themselves to join the enlightened throng? Only the most dedicated can afford the time necessary to gain experience in a recognized "fluorescence" laboratory. More ordinary mortals have recourse to reading reviews, specialized articles and older classic books and papers selected from a large and diffuse literature. This can be a hard, unrewarding slog for the newcomer, and there has long been a need for a suitable primer in this field.

Joseph Lakowicz has attempted to remedy this need by bringing within a single monograph the basic introduction, essential theory and working practice of the most important aspects of fluorescence spectroscopy, at least as encountered in the life sciences. The reader has the vicarious benefit both of what is clearly a thorough knowledge of the literature, and of the author's experience in a well-known laboratory since his treatment strongly reflects the influence of Gregorio Weber to whom the book is dedicated. This is no small recommendation in itself. Phasemodulation techniques, after two decades of undeserved and incidental eclipse by pulse-excitation, are rightly restored to pre-eminence, while the phase-dependent theory of decaying states. A final point is that it seems perverse in a book largely devoted to interactions of neutrinos to normalize spinors in a way which is singular for massless particles.

Despite its faults, this is not a bad book. There are better introductions to gauge theories, such as Gauge Theories in Particle Physics by I.J.R. Aitchison and A.J.G. Hey (Adam Hilger, 1982), and clearer discussions of the phenomenology of weak interactions, for example in L.B. Okun's Leptons and Quarks (NorthHolland, 1982) or T.D. Lee's monumental Particle Physics and Introduction to Field Theory (Harwood, 1981). However, this is the only up-to-date book on weak interactions which includes a discussion of the experiments and the data, and I would therefore advise students to read it and librarians to buy it.

C.H. Llewellyn Smith is a Reader in the Department of Theoretical Physics, University of Oxford.

method developed by the author for resolution of heterogeneous emission occupies a complete short chapter. Longer, wellillustrated sections are devoted to the fundamental fluorescence parameters and to a selection of excited-state processes implied by the "spectroscopy" in the title. As the latter suggests, the main emphasis is on fundamental aspects, while examples are taken almost entirely from protein and membrane systems.

A description of the essential instrumentation, calibration methods, common malfunctions and experimental pitfalls comes immediately after a short general introduction. This introduction, however, is barely adequate in conveying the range of molecular information potentially made accessible by appropriate strategies, and an early chance to whet appetites is rather missed. That said, the level of presentation is nicely poised between the superficial and the recondite. So that the reader has some likelihood of pursuing them, references are restricted to major benchmark publications (except for several "unpublished observations", which would have been better dealt with in the text). A useful feature, stressing the didactic intention of the author, is the inclusion of simple problems at the end of most chapters; if attempted, these oblige the reader to understand fully the main thrust of the preceding material. Correct solutions are also provided for reassurance.

Lakowicz's aim in writing this book was to satisfy the requirements of those interested in understanding the principles underlying the various fluorescence techniques encountered in contemporary research. To my mind, he has succeeded.

F.W.J. Teale is the Reader in Physical Biochemistry in the Department of Biochemistry, University of Birmingham. 